SUBJECT AREAS:

MOLECULAR

NEUROSCIENCE

TARGET IDENTIFICATION

Received

31 December 2013

Accepted

18 March 2014

Published

9 April 2014

Correspondence and requests for materials should be addressed to

M.P. (pizzi@med.

unibs.it) or S.O.

(simone.ottonello@

unipr.it)

* These authors contributed equally to this work.

$\uparrow$ Current address:

Microbiological Laboratory,

GlaxoSmithKline

Manufacturing $\mathrm{SpA}$,

Via Asolana 90

43056 - S. Polo di

Torrile (Parma), Italy.

\section{Pharmacological targeting of the} $\beta$-amyloid precursor protein intracellular domain

\author{
Caterina Branca ${ }^{1 *}$, llenia Sarnico ${ }^{*}$, Roberta Ruotolo ${ }^{3}$, Annamaria Lanzillotta ${ }^{1}$, Arturo Roberto Viscomi ${ }^{3} \uparrow$, \\ Marina Benarese' ${ }^{1}$, Vanessa Porrini ${ }^{1}{ }^{2}$, Luca Lorenzini ${ }^{4}$, Laura Calzà ${ }^{4}$, Bruno Pietro Imbimbo ${ }^{5}$, \\ Simone Ottonello ${ }^{3} \&$ Marina Pizzi ${ }^{1,2}$

\begin{abstract}
'Department of Molecular \& Translational Medicine and National Institute of Neuroscience, School of Medicine, University of Brescia, Italy, ${ }^{2}$ IRCCS, San Camillo Hospital, Venice, Italy, ${ }^{3}$ Laboratory of Functional Genomics and Protein Engineering, Biochemistry and Molecular Biology Unit, Department of Life Sciences, University of Parma, Italy, ${ }^{4}$ Department of Veterinary Medicine and Health Science, University of Bologna, Italy, ${ }^{5}$ Research and Development, Chiesi Farmaceutici, Parma, Italy.
\end{abstract}

Amyloid precursor protein (APP) intracellular domain (AICD) is a product of APP processing with transcriptional modulation activity, whose overexpression causes various Alzheimer's disease (AD)-related dysfunctions. Here we report that 1-(3',4'-dichloro-2-fluoro[1,1'-biphenyl]-4-yl)-cyclopropanecarboxylic acid) (CHF5074), a compound that favorably affects neurodegeneration, neuroinflammation and memory deficit in transgenic mouse models of $\mathrm{AD}$, interacts with the AICD and impairs its nuclear activity. In neuroglioma-APPswe cells, CHF5074 shifted APP cleavage from $A \beta_{42}$ to the less toxic $A \beta_{38}$ peptide without affecting APP-C-terminal fragment, nor APP levels. As revealed by photoaffinity labeling, CHF5074 does not interact with $\gamma$-secretase, but binds to the AICD and lowers its nuclear translocation. In vivo treatment with CHF5074 reduced AICD occupancy as well as histone $\mathrm{H} 3$ acetylation levels and transcriptional output of the AICD-target gene KAIl. The data provide new mechanistic insights on this compound, which is under clinical investigation for AD treatment/prevention, as well as on the contribution of the AICD to AD pathology.

r he main pathological features of Alzheimer disease (AD) are neuronal loss and the presence of neurofibrillary tangles together with senile plaques consisting of amyloid beta peptides $\left(A \beta_{40}\right.$ and $\left.A \beta_{42}\right)$ produced by cleavage of the amyloid precursor protein (APP) in affected brain regions $s^{1,2}$. APP processing can proceed through alternative, non-amyloidogenic or amyloidogenic pathways, initiated by either $\alpha$ - or $\beta$-secretase cleavage and leading to the production of two large soluble fragments ( $A P P \alpha$ and sAPP $\beta$ ) and two membrane-anchored C-terminal fragments, CTF $\alpha$ and CTF $\beta$, respectively. CTF processing by $\gamma$-secretase generates the harmless P3 peptide (non-amyloidogenic pathway) or $A \beta$ peptides ranging in size from 35 to 42 amino acids (amyloidogenic pathway), plus the APP intracellular domain (AICD) fragment ${ }^{3-5}$. Gamma-secretase is a multisubunit protease that catalyzes the intramembrane processing of more than 50 type-I membrane proteins ${ }^{6}$. It is composed of presenilin (PS1 or PS2), the catalytic subunit responsible for CTF processing, nicastrin (Nct) and the multipass transmembrane proteins presenilin enhancer 2 (Pen-2) and anterior pharynx-defective 1 (Aph-1) ${ }^{4,5}$. The amyloidogenic pathway has been shown to be the major source of AICD in vivo ${ }^{7,8}$.

By acting as a docking site for a heterogeneous set of adaptor proteins, the AICD has been shown to be involved in a variety of signaling processes, many of which are potentially relevant to AD pathology ${ }^{9-11}$. The best known AICD interactor is the adaptor protein Fe65, which stabilizes the AICD and promotes its nuclear transloca$\operatorname{tion}^{3,12,13}$. In the nucleus, the AICD has been reported to be present within dot-like structures also containing Fe65 and the histone acetyl-transferase Tip60 $0^{12-14}$. This multiprotein complex is involved in transcriptional activation $^{15-17}$, though the specific role(s) of the AICD in such process, its full set of partner proteins as well as the range of genes it can target are still debated ${ }^{3,18}$. The best established AICD target gene codes for the pro-apoptotic tetraspannin KAI1, also known as CD82 $2^{14,15,19,20}$.

AICD and Fe65 overexpressing mice do not show brain $A \beta$ accumulation, yet display a number of $A D$-like neuropathological features, including tau hyperphosphorylation, glycogen synthase kinase-3 $\beta$ (GSK-3 $\beta$ ) activation, working memory deficits and neuroinflammation ${ }^{9,21}$. Because AICD levels were found to be elevated in 
human $\mathrm{AD}$ brains ${ }^{9,22}$, it has been hypothesized that this C-terminal APP fragment may be causally involved in AD pathogenesis ${ }^{9,10,23,24}$. However, small-molecules able to bind the AICD and interfere with its function(s) have not been described so far.

In various transgenic mouse models of $\mathrm{AD}$, the non-steroidal antiinflammatory derivative (NSAID) CHF5074 [1-(3', $4^{\prime}$-dichloro-2fluoro[1,1'-biphenyl]-4-yl)-cyclopropanecarboxylic acid] (Fig. 1a) has been found to lower plaque burden ${ }^{25}$, tau hyperphosphorylation and GSK-3 $\beta$ levels ${ }^{26}$, and to reduce neuroinflammation and associated memory deficit ${ }^{25,27,28}$, thus acting simultaneously on multiple therapeutic AD targets. CHF5074 has also been shown to improve cognition and to reduce brain inflammation in ApoE4 patients with

a<smiles>O=C(O)C1(c2ccc(-c3ccc(Cl)c(Cl)c3)c(F)c2)CC1</smiles>

b
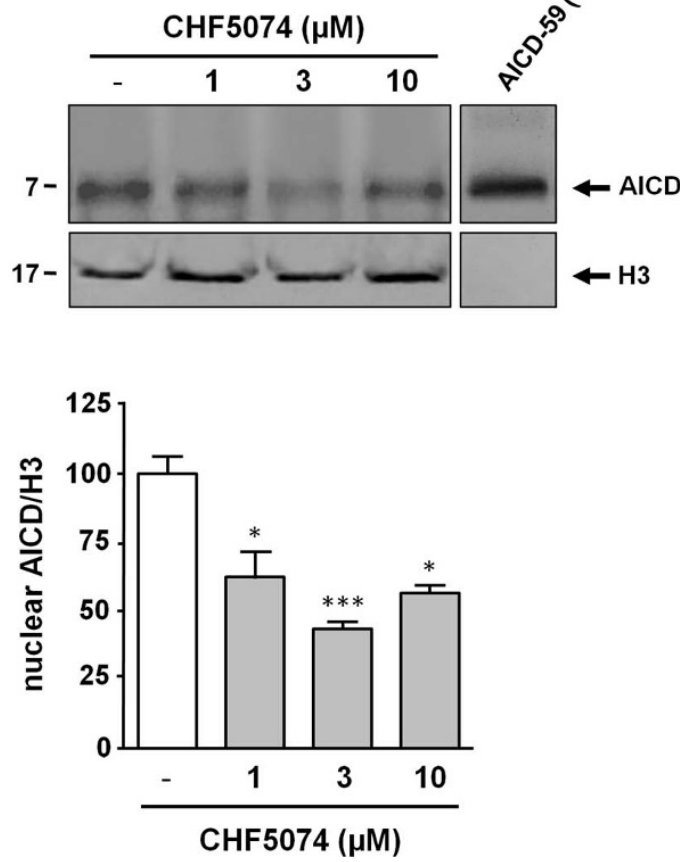

Figure $1 \mid$ CHF5074 lowers AICD nuclear levels in H4swe cells. (a) Chemical structure of CHF5074. (b) Immunoblot analysis (anti-APP C-terminal antibody) of nuclear extracts from H4swe cells treated for 18 hours with vehicle or with different concentrations of CHF5074 $(1-10 \mu \mathrm{M})$ as indicated. A single polypeptide band immunoreactive to the anti-APP C-terminal antibody was detected in nuclear templates. Synthetic AICD-59 polypeptide ( $7 \mathrm{kDa}$; shown on the right) was used as a molecular mass and electrophoretic mobility reference for these experiments (upper row). Polypeptide band from the same gel was detected with an anti-histone $\mathrm{H} 3$ antibody (lower row). Full-length blots were presented in Supplementary Figure S2. Data from multiple experiments are presented as bar plots derived from densitometric quantification of anti-APP C-terminal antibody immunoreactive bands ("test") normalized with respect to the immunoreactivity signals ("control") produced by the anti-histone $\mathrm{H} 3$ antibody. The test/control ratio for vehicle-treated samples was arbitrarily set to $100 \%$; bars are the mean \pm s.e.m. of three independent experiments $\left({ }^{*}, p<0.05\right.$; $* * *, p<0.001)$. mild cognitive impairment $(\mathrm{MCI})^{29}$. Similar to other NSAIDs, CHF5074 modulates $\gamma$-secretase activity in vitro ${ }^{30}$, although this modulatory action does not translate into a measurable effect on cerebrospinal fluid (CSF) A $\beta$ levels in humans ${ }^{31}$.

Starting from the observation that CHF5074 lowered nuclear AICD levels in neuroglioma cells expressing the Swedish mutated form of human APP (H4swe) without inhibiting, but rather modulating, $\gamma$-secretase activity, we investigated the interaction of CHF5074 with the APP substrate and the $\gamma$-secretase enzyme. As revealed by photoaffinity labeling studies, CHF5074 targets the AICD region of APP-CTF without any detectable interaction with $\gamma$-secretase. In addition to a lowered AICD nuclear translocation in H4swe cells, CHF5074 treatment of transgenic mice overexpressing the Swedish mutated form of human APP also markedly reduced AICD binding and $\mathrm{H} 3$ acetylation levels at the promoter region of the AICD target gene KAI1, with a concomitant reduction of its transcriptional output. By demonstrating for the first time the targeting of the AICD region of APP by a small-molecule compound, the data suggest a plausible mechanism that might contribute to, and explain, the broad-spectrum neuroprotective properties of CHF5074.

\section{Results}

CHF5074 reduces nuclear AICD levels in H4swe cells without inhibiting $\gamma$-secretase activity. H4swe cells are a human neuroglioma cell line producing the same K595N/M596L mutated form of APP expressed by the Tg2576 mouse model of AD that has been extensively utilized for the preclinical characterization of CHF$5074^{25,27,28,30,32,33}$. To evaluate whether CHF5074, at concentrations $(1-10 \mu \mathrm{M})$ that inhibit $\mathrm{A} \beta_{42}$ generation (see Supplementary Fig. $\mathrm{S} 1 \mathrm{a})^{30}$, can interfere with the nuclear translocation of the AICD, we examined AICD levels in nuclear extracts from treated and untreated H4swe cells. As shown in Fig. 1b (and Supplementary Fig. S2), a single band corresponding to AICD fragment was revealed by antiAPP C-terminus antibody. An 18 hour treatment caused a decrease of AICD nuclear levels, which were reduced by $60 \%$ at a CHF5074 concentration of $3 \mu \mathrm{M}$. To rule out a possible general inhibitory effect of CHF5074 on APP-CTF cleavage, that would also negatively affect AICD production, we analyzed APP processing in H4swe cell extracts with the use of fragment-specific antibodies recognizing different regions of the APP (see below). We found that exposure to CHF5074 $(1-30 \mu \mathrm{M})$ for 18 hours, neither affected CTF $\beta$ nor full-length-APP (FL-APP) levels, as detected in whole cell extracts by immunoblotting with the 6E10 antibody (Fig. 2a). Similarly, no significant changes were observed in $\operatorname{sAPP} \alpha$ or sAPP $\beta$ levels by immunoblot analysis of conditioned cell culture media performed with the anti-APP N-terminal antibody (Fig. 2b and Supplementary Fig. S3a). As further shown in Fig. 2c (and Supplementary Fig. S3b), CHF5074 also did not modify neither $\mathrm{CTF} \alpha$, nor CTF $\beta$ levels, detected by immunoblot analysis of total cell extracts with the anti-APP C-terminal antibody (see Methods for details). By contrast, a significant increase of both APP-CTFs was observed under identical experimental conditions upon treatment with the $\gamma$-secretase inhibitor $\mathrm{N}$-[N-(3,5-difluorophenacetyl)-Lalanyl]-S-phenylglycine t-butyl ester) (DAPT; $0.2 \mu \mathrm{M}$ ).

Although the anti-APP C-terminal antibody can also recognize the AICD, this fragment could not be detected in H4swe whole cell extracts under standard immunoreaction conditions. Instead, the AICD was detected in nuclear extracts (see Fig. 1b), in accordance with previous data indicating that only the AICD produced at the endosome compartment (i.e., in close proximity to the nuclear compartment) can be translocated to the nucleus, thus escaping rapid cytoplasmic degradation ${ }^{8}$. Therefore, the unchanged levels of the APP-CTFs observed in whole extracts from CHF5074-treated cells (see Fig. 2c) suggest that nuclear translocation, rather than $\gamma$-secretase-mediated generation of the AICD, is modified by CHF5074 treatment. 
a
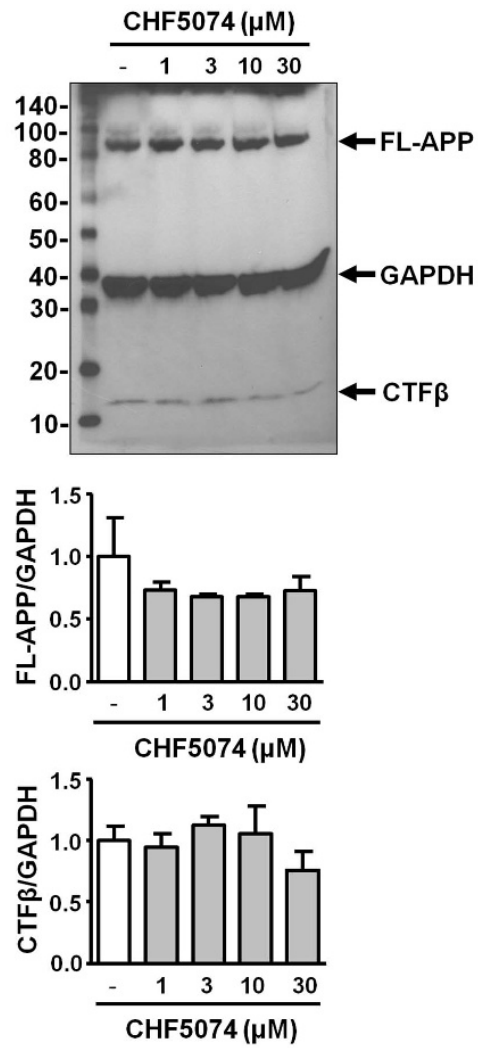

b
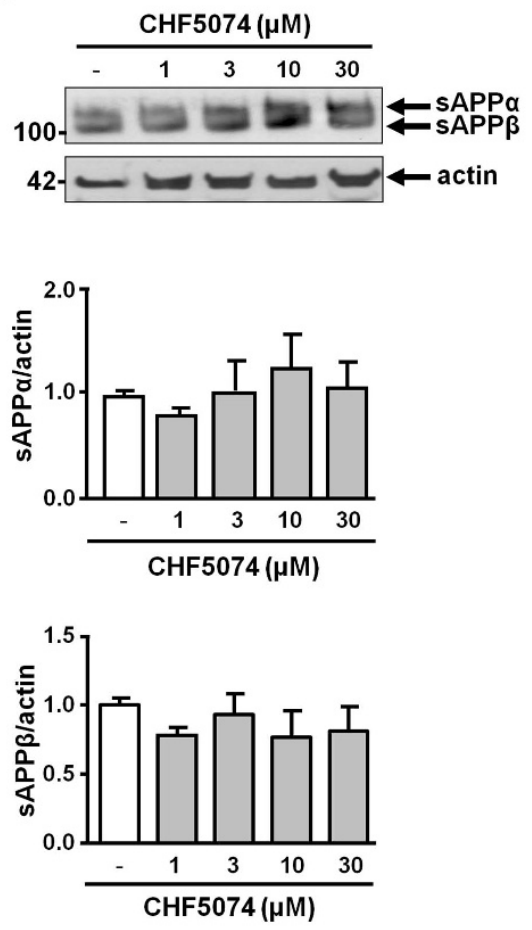

C
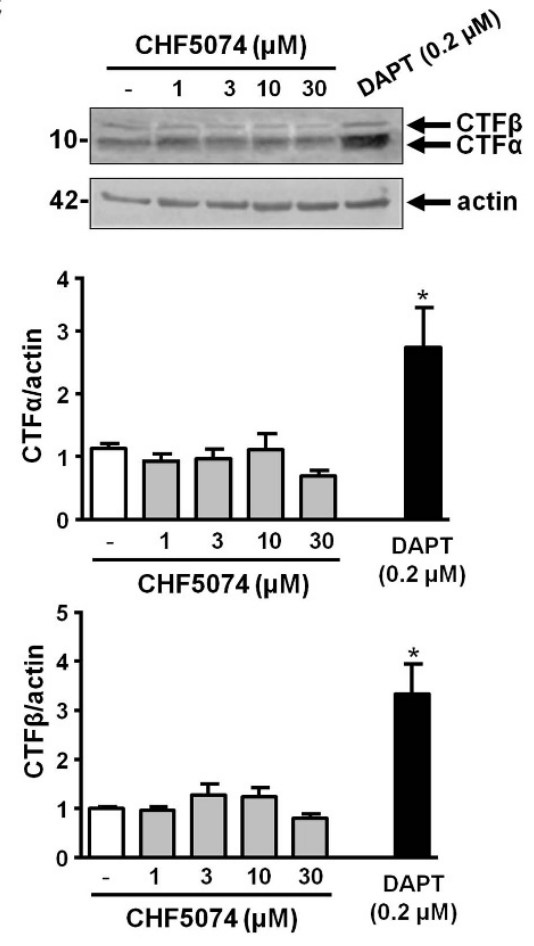

Figure $2 \mid$ APP processing in CHF5074-treated H4swe cells. (a) Immunoblot analysis of whole extracts from H4swe cells treated for 18 hours with vehicle or different concentrations of CHF5074 (1-30 $\mu \mathrm{M})$. CTF $\beta$ and FL-APP, whose levels did not appreciably change upon treatment with CHF5074, were detected with the 6E10 antibody; GAPDH, measured in the same blot with an anti-GAPDH antibody, was used as a loading control. (b) Immunoblot analysis as in (a) performed with the anti-APP N-terminal antibody in culture medium of H4swe cells treated for 18 hours with vehicle or different concentration of CHF5074 (1-30 $\mu \mathrm{M})$. Similar sAPP fragment levels were detected in all samples regardless of CHF5074 treatment. sAPP fragments in the medium were normalized to the actin content detected in the corresponding releasing cells. Full-length blots were presented in Supplementary Figure S3a c) Immunoblot analysis, performed with the anti-APP C-terminal antibody of extracts from H4swe cells treated with vehicle, CHF5074 (1-30 $\mu \mathrm{M})$ or DAPT $(0.2 \mu \mathrm{M})$ for 18 hours. DAPT, but not CHF5074, increases CTF $\alpha$ and $\beta$ levels $(*, p<0.05)$. Full-length blots were presented in Supplementary Figure S3b. Bar plots, shown below each gel image, were obtained by densitometric analysis of immunoblot data normalized with respect to the immunoreactivity signals detected by control antibodies ( GAPDH, panel a; actin, panels b and c). The test/control ratio for vehicle-treated samples was set to 1.0 ; bars represent the mean \pm s.e.m. of three independent experiments.

In accordance with previous studies ${ }^{30}$, ELISA analysis of secreted $A \beta$ peptides showed that CHF5074 preferentially inhibits $A \beta_{42}$ compared to $\mathrm{A} \beta_{40}$ production. $\mathrm{IC}_{50}$ values were $2.2 \mu \mathrm{M}$ and $24 \mu \mathrm{M}$ for $\mathrm{A} \beta_{42}$ and $\mathrm{A} \beta_{40}$, respectively (Supplementary Fig. S1a). We also found that the differential effect on $A \beta_{40}$ and $A \beta_{42}$ levels, was accompanied by an enhanced production of the non-toxic $A \beta_{38}$ peptide. The $\mathrm{EC}_{50}$ for the stimulatory effect of CHF5074 on $\mathrm{A} \beta_{38}$ production $(2.2 \mu \mathrm{M})$ was identical to that causing half-inhibition of $A \beta_{42}$ secretion. The resulting curve was bell-shaped and peaked at a $10 \mu \mathrm{M}$ concentration of CHF5074 (Supplementary Fig. S1b).

Photoaffinity labeling analysis of the CHF5074-APP interaction. Photoaffinity labeling studies, using a a photoactivatable benzophenonebiotin derivative of CHF5074 (CHF5074-BpB; Fig. 3a), were then carried out to investigate the interaction of CHF5074 with APP-CTF and the multiprotein $\gamma$-secretase complex.

As a prerequisite for this analysis, we first verified the functionality of chemically modified CHF5074-BpB. As shown in Fig. 3, the $\gamma$ secretase modulation activity of CHF5074-BpB resembles that of the parent compound (see also Supplementary Fig. S1a and S1b). Compared to CHF5074, CHF5074-BpB was somewhat more potent in reducing $A \beta_{42}$ than $A \beta_{40}$ generation (Fig. 3b), but retained a similar capacity to enhance $A \beta_{38}$ production (Fig. $3 c$ ). Likewise, CHF5074-BpB did not modify the cellular levels of neither the CTFs, nor FL-APP (Fig. 3d and Supplementary Fig. S4), and even at high concentrations (up to $100 \mu \mathrm{M}$ ) it did not appreciably affect cell viability (Fig. 3e).

We thus proceeded with in vitro photolabeling by incubating H4swe membrane protein fractions in the presence of CHF5074$\mathrm{BpB}(3 \mu \mathrm{M})$, followed by streptavidin-mediated precipitation and immunoblot analysis using the 6E10 antibody. As shown in Fig. 4a (and Supplementary Fig. S5a), CHF5074-BpB-cross-linked proteins bound by steptavidin beads, included FL-APP and the CTF $\beta$ fragment. A lower immune-signal, especially for FL-APP, was observed when labeling was performed on whole cell lysates rather than on membrane fractions (Fig. 4a). With both samples APP-CTF displayed a higher reactivity, which was significantly quenched $(\sim 60 \%)$ by preincubation with a 33 -fold molar excess of unmodified CHF5074 (Fig. 4b and Supplementary Fig. S5b, cf. the two right-most lanes). Importantly, parallel photolabeling/immunoblot experiments performed on H4swe membranes under both denaturing (1\% Nonidet-P40 - NP40) and native conditions, using antibodies targeting four $\gamma$-secretase subunits (PS1, PS2, Nct and Pen-2) did not reveal any interaction between CHF5074-BpB and the $\gamma$-secretase enzyme complex (Fig. 4c).

CHF5074 binds to the intracellular domain of APP. To gain more detailed information on the APP binding site of CHF5074, we examined CHF5074-BpB reactivity with two synthetic polypeptides corresponding to the $A \beta_{42}$ and AICD-59 fragments of APP 


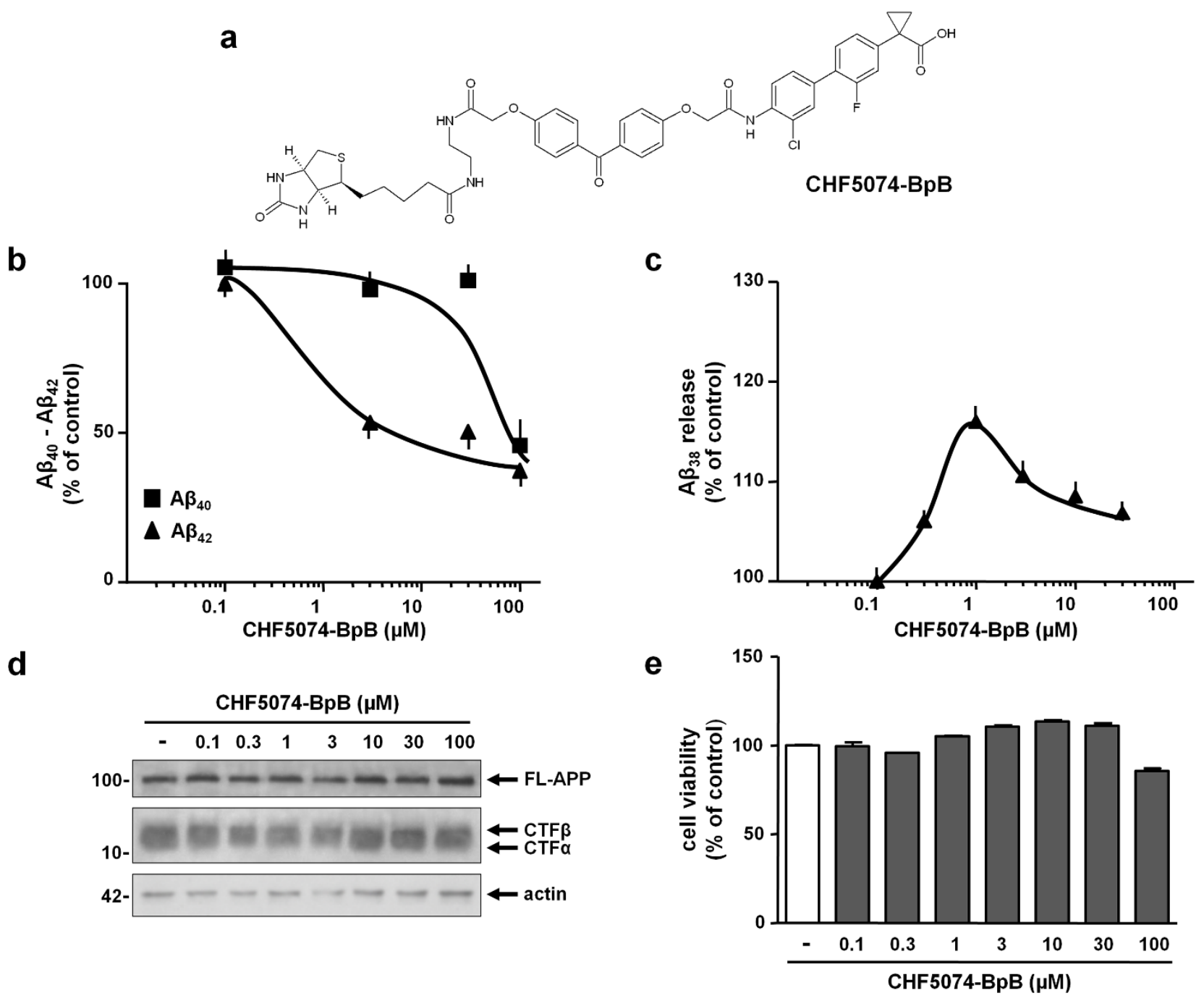

Figure $3 \mid$ The photoactivatable CHF5074-BpB derivative retains the $\gamma$-secretase modulatory properties of the parent compound. (a) Chemical structure of the benzophenone-biotin (BpB) derivative of CHF5074, whose synthesis is described in Supplementary Methods. Similar to the parent compound, CHF5074-BpB reduced $\mathrm{A} \beta_{40}$ and $\mathrm{A} \beta_{42}$ secretion (b) and increased $\mathrm{A} \beta_{38}$ production (cf. data reported in Supplementary Fig. S1a and S1b) (c). $A \beta$ peptide release was evaluated by ELISAs performed on conditioned culture media from $\mathrm{H} 4$ swe cells exposed to the indicated concentrations of CHF5074-BpB $(0.1-100 \mu \mathrm{M})$ for 18 hours. Data are percentages of control \pm s.e.m. from three independent experiments. (d) Representative results obtained from immunoblot analysis (anti-APP C-terminus and control anti-actin antibodies as in Fig. 2c) of extracts from H4swe cells treated with vehicle or CHF5074-BpB (1-100 $\mu \mathrm{M})$. Full-length blots were presented in Supplementary Figure S4. (e) Cell viability, assayed with the lactate dehydrogenase $(\mathrm{LDH})$ release assay, was not appreciably affected by CHF5074-BpB at any of the tested concentrations; data are presented as percentages of control \pm s.e.m. from three independent experiments.

and with a recombinant glutathione S-transferase-CTF $\alpha$ (GST$\mathrm{CTF} \alpha$ ) fusion polypeptide (see Fig. 5a). Each polypeptide (20 pmol) was individually incubated with CHF5074-BpB $(3 \mu \mathrm{M})$, alone and in the presence of an excess of unmodified CHF5074. As shown in Fig. 5b (upper panels), both AICD-59 and GST-CTF $\alpha$, but neither $\mathrm{A} \beta_{42}$ peptide, nor the GST carrier protein alone, were detected with IrDye-labeled streptavidin following treatment with CHF5074-BpB and the resulting signals were both quenched $(\sim 30 \%$ and $70 \%$, respectively) by addition of the unmodified compound. Comparable results in terms of photolabeling selectivity were obtained with CHF5074-BpB concentrations as low as $0.5 \mu \mathrm{M}$ (data not shown) and a similar lack of CHF5074-BpB reactivity with $A \beta$ was observed with monomeric (Fig. 5b), as well as oligomeric (Supplementary Fig. S6) $A \beta_{42}$. Altogether, the above results point to the specific binding of CHF5074 to the AICD, a C-terminal region of APP downstream to the cleavage site of $\gamma$-secretase.

In vivo treatment with $\mathrm{CHF} 5074$ reduces $\mathrm{AICD}$ occupancy, histone acetylation levels and transcriptional activity of an AICD target gene. We next evaluated whether CHF5074, in addition to its lowering effect on nuclear AICD levels in a cell-based model of $\mathrm{AD}$ (Fig. 1b), could also affect nuclear AICD functionality and target gene promoter occupancy in vivo. To this end, we performed a chromatin immunoprecipitation (ChIP) analysis on brain cortices from $\operatorname{Tg} 2576$ mice treated with either CHF5074-supplemented diet (375 ppm; $\approx 60 \mathrm{mg} / \mathrm{kg} /$ day) or standard diet for 4 weeks. The choice of this treatment protocol was based on previous preclinical data showing full reversal of recognition memory and a marked amelioration of various AD-related neuropathological features, in Tg2576 mice orally treated with the same dose of CHF5074 for 4 weeks ${ }^{32}$. The tetraspanin-encoding KAI1 gene, a broadly supported AICDtarget ${ }^{14,15,20}$ previously subjected also to ChIP analysis ${ }^{15,20}$, was used to test the effect of CHF5074 treatment. To this end, cross-linked chromatin from brain cortices of treated and untreated animals was separately immunoprecipitated with the anti-APP C-terminal antibody, an anti-acetylated histone $\mathrm{H} 3$ antibody, and an unrelated, control antibody. As revealed by quantitative RealTime-PCR analysis of immunoprecipitated DNA (Fig. 6a), treatment with CHF5074 
a

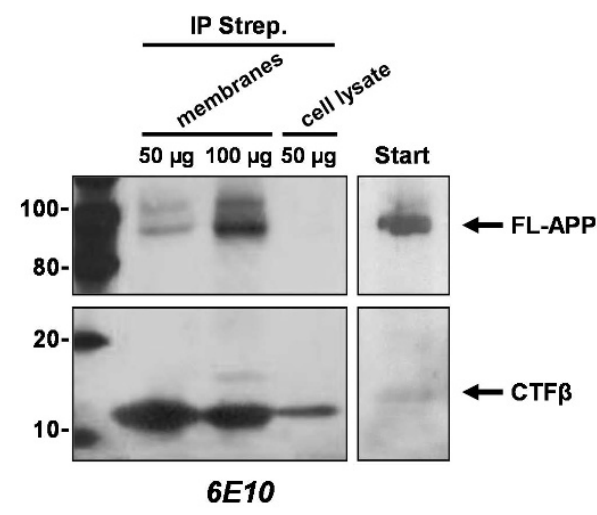

b

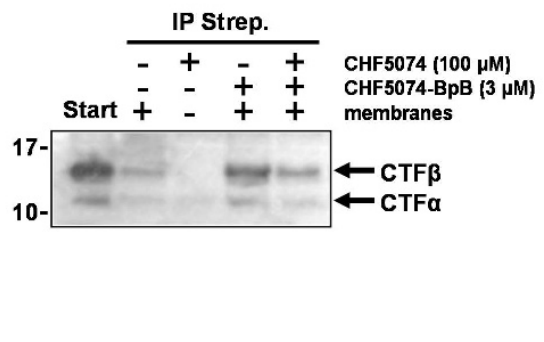

C

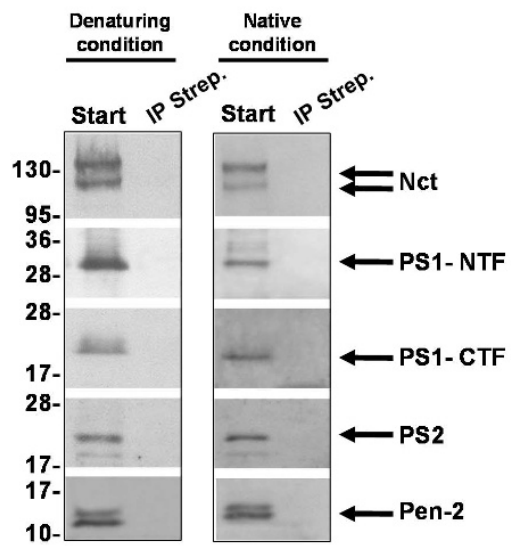

Figure 4 | Photoaffinity labeling analysis of CHF5074-BpB-treated H4swe cells. (a) Photoaffinity labeling of FL-APP and CTF $\beta$ in membrane fractions and total lysates from H4swe cells incubated with CHF5074-BpB $(3 \mu \mathrm{M})$ and exposed to ultraviolet light (350 nm, 30 minutes), prior to streptavidin precipitation $(I P)$, gel-fractionation and immunoblot analysis with the $6 \mathrm{E} 10$ monoclonal antibody. Immunoreactivity of naïve membrane fractions (i.e., membranes not treated with CHF5074-BpB and not subjected to streptavidin precipitation) is shown on the right ("start"). Full-length blots were presented in Supplementary Figure S5a. (b) Reduced CHF5074-BpB labeling of APP-C-terminal fragments upon preincubation with unmodified CHF5074 $(100 \mu \mathrm{M})$. Labeling was performed on membrane fractions, followed by streptavidin precipitation, gel-fractionation and immunoblot analysis with the anti-APP C-terminal antibody (see legend to panel a for further details). Specific labeling by CHF5074-BpB was detected over the background signals observed when omitting the photoprobe (lane 2) or the membranes (lane 3) from the reaction mixture. Full-length blots are presented in Supplementary Figure S5b. (c) Immunoblot analysis of CHF5074-BpB-treated membranes as in (a), performed with antibodies directed against the indicated $\gamma$-secretase subunits: nicastrin, Nct; presenilin-1 N-terminal fragment, PS1-NTF; presenilin-1 C-terminal fragment, PS1-CTF; presenilin-2, PS2; presenilin enhancer-2, Pen-2 (see legend to panel a for further details). Lack of photolabeling was observed even when the membranes were exposed to the CHF5074-BpB photoprobe prior to detergent solubilization ("native conditions"). Cropped blot images (derived from ten different experiments) were shown.

was associated to an $79 \%$ reduction of AICD occupancy and an $72 \%$ decrease of histone $\mathrm{H} 3$ acetylation at the KAI1 promoter. As further shown in Fig. 6b, these changes were accompanied by a significant reduction of KAII transcripts. These data suggest that, in addition to nuclear translocation, CHF5074 binding to the AICD interferes with nuclear transactions (i.e., promoter binding, recruitment of histone acetyl-transferase components such as Tip60 and target gene transcriptional output) mediated by the intracellular C-terminal fragment of APP.

\section{Discussion}

The main finding of this study is the peculiar ability of the antiinflammatory derivative CHF5074 to reduce nuclear translocation and activity of the APP intracellular domain by binding to the AICD peptide without altering its $\gamma$-secretase-mediated generation.

In accordance with previous data ${ }^{30}$, CHF5074 preferentially reduced $A \beta_{42}$, compared to $A \beta_{40}$, levels and increased production of the non-toxic $A \beta_{38}$ peptide in H4swe neuroglioma cells, the cellular counterpart of the Tg2576 mouse model of AD. This suggests that CHF5074 does not inhibit APP processing, but rather shifts the site of APP-CTF cleavage. Indeed, CHF5074 did not increase APP-CTF levels, as observed with the $\gamma$-secretase inhibitor DAPT (Fig. 2c; but see also $\left.^{34}\right)$, and neither affected soluble APP fragment levels, nor the levels of FL-APP, indicating the lack of any effect on $\alpha$-secretase or $\beta$ secretase activity.

Using a photoaffinity labeling approach, we found that CHF5074 binds to the AICD region of the APP-CTF substrate, but not to the $\gamma$ secretase complex. Binding selectivity was inferred from competition of unmodified CHF5074 on the binding of CHF5074-BpB to the AICD, which took place at concentrations 30- to 100-fold lower than those employed for photoaffinity labeling with $\mathrm{BpB}$ derivatives of $1^{\text {st }}$ generation $\gamma$-secretase modulators ${ }^{35}$. In line with recent models for sequential cleavage by $\gamma$-secretase ${ }^{36,37}$, we hypothesize that CHF5074 binding to the AICD region of APP may influence $\gamma$-secretase processivity by altering the dynamics and/or the stereochemistry of interaction between the enzyme and its substrate.
CHF5074 lowered AICD occupancy, H3 acetylation levels and transcriptional output of the target gene KAII, suggesting that CHF5074 binding interferes not only with the nuclear translocation, but also with the nuclear activity (i.e., promoter binding and co-activator recruitment) of the AICD. The KAI1 gene codes for a proapototic tetraspanin and is one of the best established AICD targets $^{14,15,19,20}$. Indeed, neuronal apoptosis is considered a common feature of $\mathrm{AD}$ and the first cellular alteration that was shown to be positively regulated by the $\mathrm{AICD}^{22,38}$; an alteration that could be prevented by blocking AICD nuclear translocation ${ }^{38}$ as well as by artificially weakening the AICD-Fe65 interaction ${ }^{17}$.

Given the reduction of nuclear AICD levels brought about by CHF5074 and the well-known AICD-stabilizing effect of the Fe65 adaptor (reviewed by ${ }^{3,23}$ ), it is tempting to speculate that CHF5074 binding to APP-CTF may hinder the AICD-Fe65 interaction. This would promote cytoplasmic instability and a reduced nuclear translocation of the AICD, ultimately leading to a decreased promoter occupancy. Other, not mutually exclusive hypotheses are also possible, however. For example, interference of CHF5074 with the APPCTF-Fe65 interaction might be sufficient to enhance cytoplasmic AICD degradation and to lower the nuclear translocation/activity of Fe $65^{39}$. The same interaction, which involves the YENPTY region of the AICD (aa. 682-687), has been reported to promote the amyloidogenic pathway thus increasing $A \beta$ production, an effect that could be blocked by mutation (Y682G) of the AICD Tyr682 residue ${ }^{40}$ as well as by artificial silencing of $\mathrm{Fe} 65^{41}$. Furthermore, reversion of various neuropathological features of $\mathrm{AD}$, including astrogliosis and cognitive impairment, has been reported in $\mathrm{Tg}-\mathrm{AD}$ mice bearing a D664A mutation in the $\mathrm{AICD}^{42}$.

Also notable is the overlap between the positive effects brought about by CHF5074 treatment in various transgenic mouse models of $\mathrm{AD}^{25-28,32,43}$ and the specific $\mathrm{AD}$-related alterations associated with AICD/Fe65 overproduction. The latter, in addition to tau hyperphosphorylation/aggregation and cognitive impairment ${ }^{9}$, include an altered cytoskeleton dynamics ${ }^{44}$, impaired neurogenesis and neuroinflammation ${ }^{21}$. All the above alterations are reverted to varying 


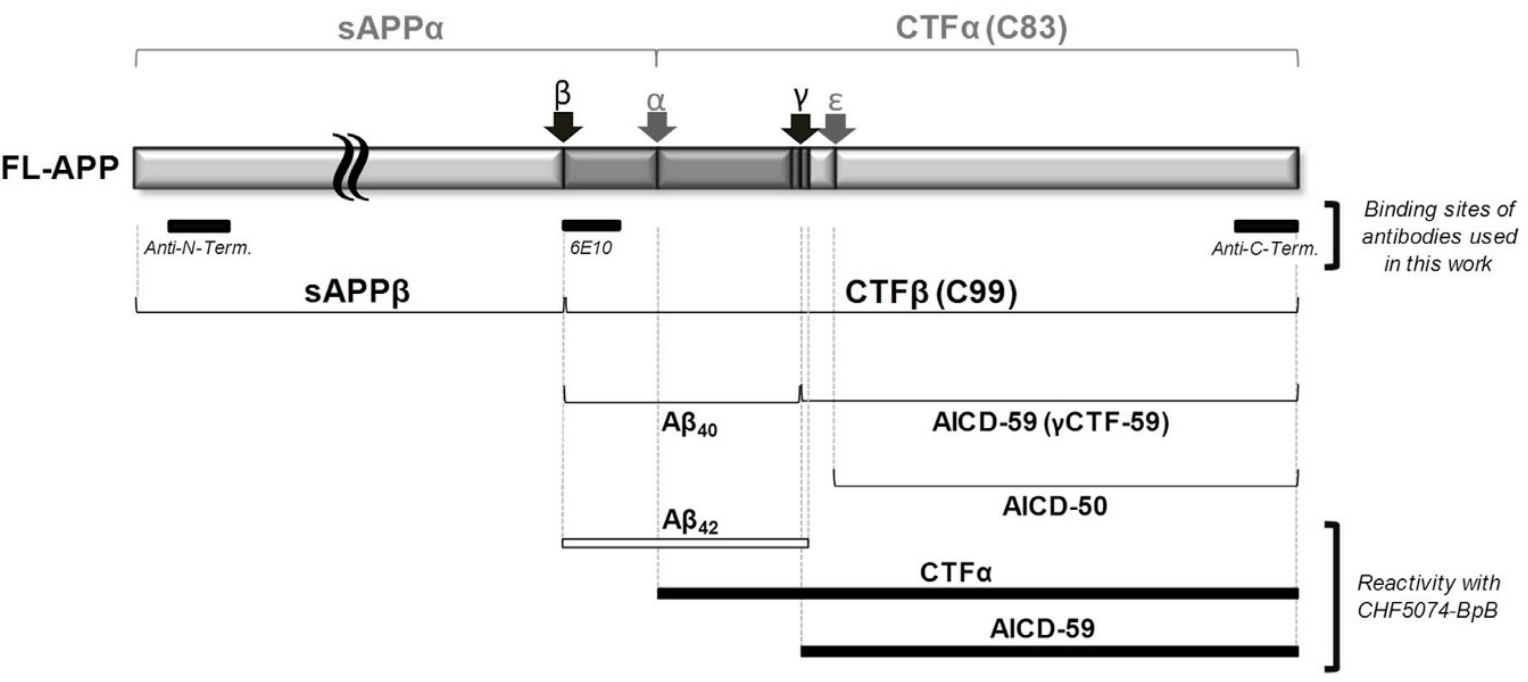

b

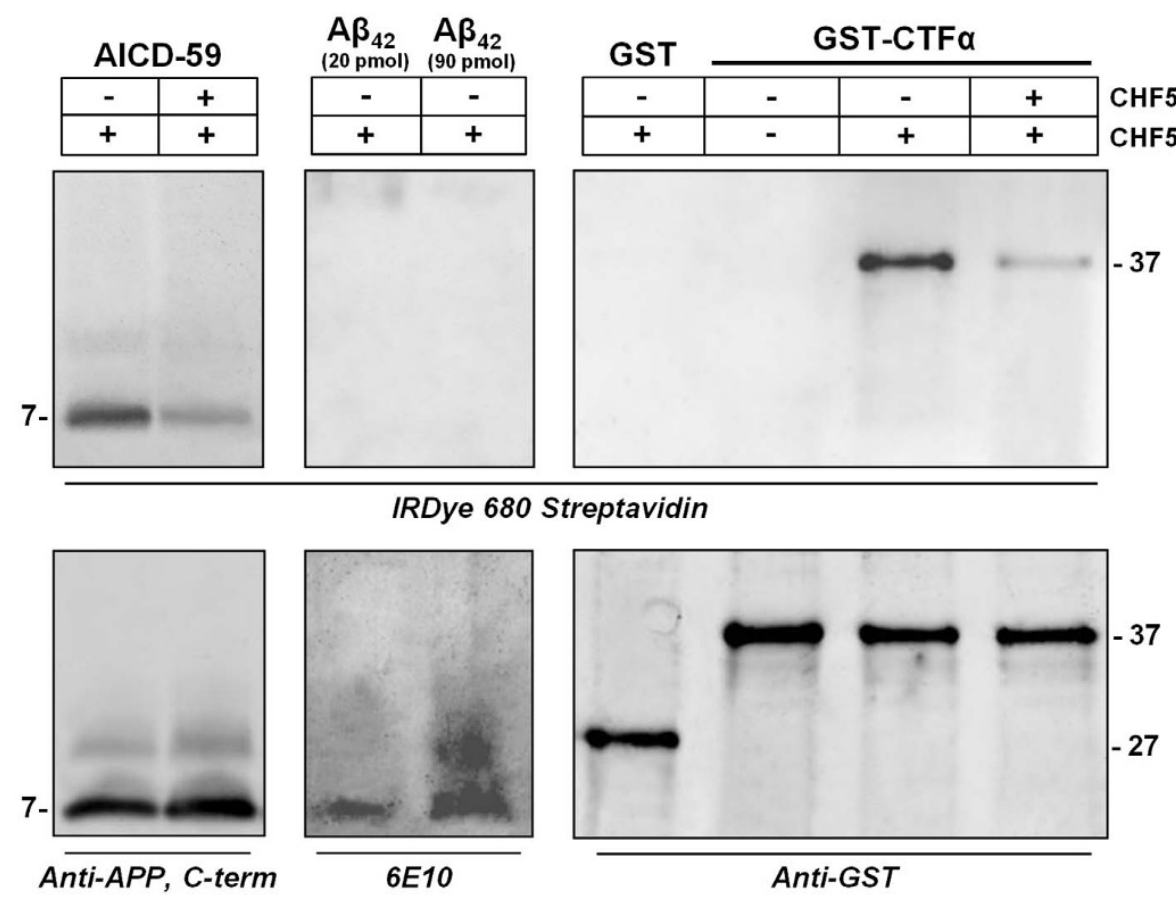

Figure $5 \mid$ Photoaffinity labeling analysis of specific APP fragments. (a) Schematic representation of APP processing products, including the binding sites of the antibodies utilized in this work and the APP fragments that were subjected to photolabeling analysis: the CHF5074-BpB-reactive CTF $\alpha$ and AICD-59 fragments (black bars) and the non-reactive $\mathrm{A} \beta_{42}$ peptide (white bar). (b) Photoaffinity labeling profiles (upper panels) obtained after incubation with $(+)$ or without $(-)$ CHF5074-BpB $(3 \mu \mathrm{M})$ of the synthetic AICD-59 $(20$ pmol, left panel $)$ and A $\beta_{42}(20$ and 90 pmol, central panel $)$ peptides, and the empty glutathione S-transferase carrier (GST) and the recombinant GST-CTF $\alpha$ fusion polypeptide (20 pmol, right panel). CHF5074-BpB reactive products were detected with IrDye 680-conjugated streptavidin. Labeling competition experiments were performed by preincubating 20 pmol of the AICD-59 peptide (left panel) or the GST-CTF $\alpha$ fusion polypeptide (right panel) in the presence $(+)$ of unmodified CHF5074 (100 $\mu \mathrm{M})$, followed by exposure to $3 \mu \mathrm{M}$ CHF5074-BpB. Parallel immunoblot analyses performed with the anti-APP C-terminal antibody (AICD-59), the 6E10 antibody (A $\beta_{42}$ ) and with an antibody directed against GST (GST and GST-CTF $\alpha$ ), served as controls to verify the input of the various (poly)peptides utilized for these experiments (lower panels). Cropped gel images are shown and the gels were run under the same experimental conditions.

extents and with a high degree of specificity compared to other NSAIDs, by CHF5074 $25-28,32,43$. In addition, recent studies indicate the ability of CHF5074 to positively affect CSF biomarkers of neuroinflammation in MCI subjects ${ }^{29}$, further suggesting that this compound may also act as a microglial modulator capable of reducing pro-inflammatory activity, while promoting the alternative antiinflammatory and phagocytic state of microglial cells (Porrini et al., AD/PD Conference 2013).

Despite increasing evidence pointing to a causal relationship between AICD overproduction and $\mathrm{AD}$, and the fact that increased
AICD levels have been detected in human AD brain ${ }^{9,22}$, data suggesting a beneficial role of this polypeptide, at least under certain conditions, have also been reported ${ }^{45}$. Moreover, another strongly supported AICD target gene, codes for the A $\beta$-degrading enzyme neprilysin ${ }^{24,46}$. Therefore, the borderline between physiological and neuropathological effects of the AICD is likely to be quite narrow and finely tuned, with a shift toward neuropathology upon massive accumulation through sustained activation of the amyloidogenic pathway.

In conclusion, the present findings provide an extended mechanistic background for the multiple effects of CHF5074. To our 
a

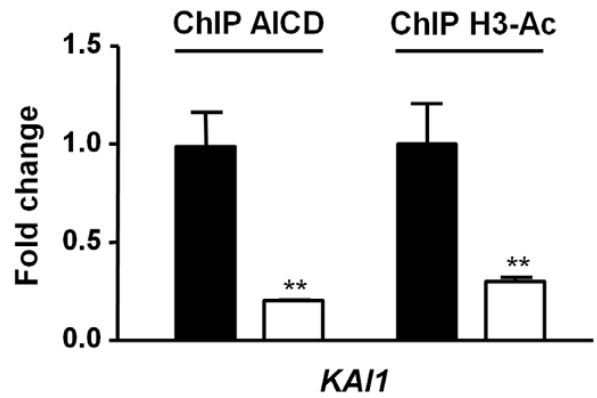

b

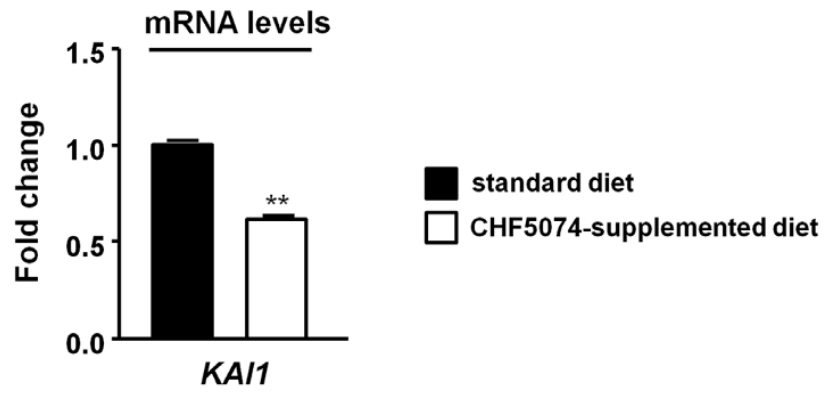

Figure 6 Effect of in vivo CHF5074 treatment on AICD occupancy, histone H3 acetylation and transcriptional activity of the KAI1 gene. (a) Chromatin immunoprecipitation assays performed on brain cortices from Tg2576 mice treated for 4 weeks with standard (black bars) or CHF5074supplemented diet ( $375 \mathrm{ppm} ; \approx 60 \mathrm{mg} / \mathrm{kg} /$ day; white bars) using anti-APP C-terminus and anti-acetyl H3 as test antibodies and an anti-IgG as a negative control antibody. DNA extracted from immunoprecipitated chromatin was analyzed by qRT-PCR, setting $\mathrm{C}_{\mathrm{T}}$ values relative to untreated samples to 1.0 (see 'Methods' for details). (b) KAI1 mRNA levels determined by qRT-PCR analysis of reverse-transcribed total RNA extracted from brain cortices of Tg2576 mice fed on standard (black bars) and CHF5074-supplemented (white bars) diet as above. Data are presented as relative values determined with the comparative $\mathrm{C}_{\mathrm{T}}$ method using $\beta$-actin as house-keeping gene reference; bars are the mean \pm s.e.m. of three independent experiments $(* *, p<0.01)$.

knowledge, CHF5074 is the first AICD-targeting small-molecule to be described and we believe that the availability of this compound will contribute to a better understanding of both the physiological role(s) of the AICD as well as its involvement in AD pathology.

\section{Methods}

Cell culture. H4swe were seeded at a density of $1.25 \times 10^{5} \mathrm{cells} / \mathrm{cm}^{2}$ in $21 \mathrm{~cm}^{2}$ culture dishes (Nunc-VWR) in Opti-MEM culture medium (Life Technologies) containing $10 \%$ fetal bovine serum and grown to confluence in a $5 \% \mathrm{CO}_{2}$ and $95 \%$ air humidified atmosphere. Cells were then incubated in Opti-MEM without serum and exposed for 18 hours to either 0.1-30 $\mu \mathrm{M}$ CHF5074 (Figure 1a), 0.1-100 $\mu \mathrm{M}$ CHF5074-BpB (Figure $3 \mathrm{a}$ ) or $0.2 \mu \mathrm{M}$ DAPT (all provided by Chiesi Farmaceutici, Parma, Italy) dissolved in dimethyl sulfoxide (DMSO; $0,1 \%$ final concentration), or to the DMSO vehicle alone. The culture medium was collected and used for ELISA and cytotoxicity assays, followed by membrane and nuclear protein extraction for immunoblot/ photoaffinity labeling.

A $\boldsymbol{\beta}$ measurements in $\mathbf{H} 4$ swe culture medium. H4swe cells were exposed for 18 hours to increasing concentrations of CHF5074 $(0.1-30 \mu \mathrm{M})$ or CHF5074-BpB $(0.1-100 \mu \mathrm{M})$ in culture medium without serum. At the end of treatment, $A \beta_{40}, A \beta_{42}$ and $A \beta_{38}$ were measured in a fixed volume $(25 \mu \mathrm{l})$ of culture medium supplemented with protease inhibitors (Sigma-Aldrich). $A \beta_{40}$ and $A \beta_{42}$ levels in the culture medium were determined with commercial ELISA kits (Life Technologies). A modification of the ELISA kit for $A \beta_{40}$ was used to measure $A \beta_{38}$ levels. A monoclonal antibody specific for the $\mathrm{N}$-terminus of human $A \beta$ was coated onto the wells of microtiter strips. Culture medium samples were then incubated with a rabbit antibody specific for the C-termini of the 1-40, 1-42 and 1-38 A $\beta$ peptides (Covance), followed by detection of bound antibodies with a horseradish peroxidase (HRP)-conjugated antirabbit antibody.

Extract preparation and immunoblot analysis. H4swe cells were harvested in $100 \mu \mathrm{l}$ of lysis buffer, sonicated for 30 seconds at full power (SONOPULS, titanium microtip MS-72) and centrifuged at $11,000 \mathrm{~g}$ for 20 minutes at $4{ }^{\circ} \mathrm{C}$. Total extracts ( $25 \mu \mathrm{g}$ total protein/sample) or $25 \mu \mathrm{l}$ of culture medium were dissolved in sample buffer and fractionated by electrophoresis on $4-12 \%$ gradient polyacrylamide-SDS gels. The anti-APP (A $\beta$ amino acids 1-16) monoclonal antibody 6E10 (SIG-39320, Covance), an anti-APP C-terminus (A8717, Sigma-Aldrich) or an anti-APP N-terminus (146132 eBioscience) antibody were used for detection. Antiglyceraldehyde 3-phosphate dehydrogenase (GAPDH - MAB374, Millipore), anti- $\beta$-actin (A5060, SigmaAldrich) and anti-histone H3 (9715, Cell Signaling Technology, CST) antibodies served as loading controls.

For nuclear protein isolation, H4swe cells were scraped into $1.5 \mathrm{ml}$ of ice-cold phosphate buffered saline (PBS). Cells were pelleted and resuspended in ice-cold buffer A [10 mM HEPES-KOH pH 7.9, $1.5 \mathrm{mM} \mathrm{MgCl}_{2}, 10 \mathrm{mM} \mathrm{KCl}, 0.5 \mathrm{mM}$ dithiothreitol, $0.2 \mathrm{mM}$ phenylmethylsulfonyl fluoride - PMSF]. Cells were allowed to swell on ice for 10 minutes, vortexed for 10 seconds and centrifuged $(18,000 \mathrm{~g}, 1$ minute at $\left.4{ }^{\circ} \mathrm{C}\right)$. The pellet was resuspended in ice-cold buffer B $(20 \mathrm{mM}$ HEPES$\mathrm{KOH}$ pH 7.9, 25\% glycerol, $0.42 \mathrm{M} \mathrm{NaCl}, 1.5 \mathrm{mM} \mathrm{MgCl}_{2}, 0.2 \mathrm{mM}$ EDTA, $0.5 \mathrm{mM}$ dithiothreitol, $0.2 \mathrm{mM}$ PMSF) and incubated on ice for 20 minutes for high-salt extraction. Cellular debris was removed by centrifugation $(18,000 \mathrm{~g}, 2$ minutes at $4^{\circ} \mathrm{C}$ ) and the supernatant was stored at $-80^{\circ} \mathrm{C}$. Immunoblot analysis of the AICD was performed according to ${ }^{47}$ using the anti-APP C-terminal antibody; the synthetic AICD-59 peptide (\#018-710, Phoenix Pharmaceuticals) served as a size reference and positive control for these experiments.
Cell membrane isolation. H4swe cells from a confluent $10 \mathrm{~cm}$ dish were collected in $500 \mu$ lof protease/phosphatase inhibitors-supplemented, ice-cold buffer C $(320 \mathrm{mM}$ sucrose, $1 \mathrm{mM}$ HEPES, $1 \mathrm{mM} \mathrm{MgCl}, 10 \mathrm{mM} \mathrm{NaHCO}_{3}, \mathrm{pH} 7.4$ ) and sonicated. Homogenates were centrifuged at $13,000 \mathrm{~g}$ for 15 minutes and membrane-containing pellets were resuspended in $300 \mu \mathrm{l}$ of ice-cold buffer D $(50 \mathrm{mM} \mathrm{NaCl}, 30 \mathrm{mM}$ triethanolamine, $50 \mathrm{mM}$ NaF, $5 \mathrm{mM}$ EGTA, $5 \mathrm{mM}$ EDTA, $10 \mathrm{mM}$ phosphonitrophenylphosphate, $50 \mu \mathrm{M}$ phenylarsine-oxide, $1 \mathrm{mM}$ benzamide, $1 \mathrm{mM} \mathrm{N}$ ethylmaleimide, $1 \mathrm{mM}$ Na-tetrathionate, $1 \%$ NP40). Membrane fractions for $\gamma$-secretase analysis were also prepared under native conditions ${ }^{48}$ by omitting the Brij35/Lubrol WX extraction step, diluted three-fold with protease inhibitorsupplemented resuspension buffer $(66.7 \mathrm{mM}$-aminocaproic acid, $1 \mathrm{mM}$ EDTA $10 \mathrm{mM}$ DTT and $50 \mathrm{mM}$ BisTris- $\mathrm{HCl}, \mathrm{pH}$ 7.0) and cross-linked with the photoprobe prior to detergent (NP-40) solubilization.

Photoaffinity labeling with CHF5074-BpB. Total cell lysates or membrane fractions (50 $\mu \mathrm{g}$ total protein/sample) were pre-incubated for 15 minutes at $37^{\circ} \mathrm{C}$ in the dark with CHF5074-BpB ( $3 \mu \mathrm{M}$; see Supplementary Methods) and then exposed to ultraviolet light $(350 \mathrm{~nm})$ for 30 minutes as described ${ }^{35}$. For competition experiments, membrane fractions were preincubated for 15 minutes with the parent compound $(100 \mu \mathrm{M})$ prior to the addition of CHF5074-BpB. Labeled samples were analyzed by SDS-PAGE after precipitation with streptavidin magnetic beads (Thermo Fisher Scientific). After blotting and antibody reaction (6E10 mAb $1: 250$ for full-length APP and APP-CTF $\beta$; anti-APP C-terminal antibody $1: 200$ for APP CTF $\alpha$ and $\beta$ ), immunoreactive proteins were detected by HRP chemiluminescence (ECL-Plus, GE-Healthcare). Gamma-secretase subunits were detected with antinicastrin $(1: 1,000$; Cell Signaling Technology); anti-PS1 N-terminus $(1: 250$; Santa Cruz Biotechnology) and C-terminus (1:1,000; CST); anti-PS2 (1:1,000; CST); and anti-Pen2 (diluted $1: 1,000$; CST) antibodies.

Photoaffinity labeling of isolated peptides was carried out by incubating CHF5074$\mathrm{BpB}(3 \mu \mathrm{M})$, directly or after preincubation in the presence of the parent compound $(100 \mu \mathrm{M})$, with $20 \mathrm{pmol}$ of AICD-59 (Phoenix Pharmaceuticals) and A $\beta_{42}$ (EZBiolab) as well as with equimolar amounts of the recombinant CTF $\alpha$ polypeptide (83 aa.). The latter was produced in Escherichia coli as a fusion protein with glutathione S-transferase (GST) (pGEX-4T-2 vector; GE-Healthcare) and purified/ solubilized from bacterial lysates as described ${ }^{49}$. After photolabeling in $50 \mathrm{mM} \mathrm{MES}$, $0.1 \mathrm{M} \mathrm{NaCl}, \mathrm{pH} 6.0$ as described above, CHF5074-BpB-treated samples were fractionated by SDS-PAGE on Criterion TGX-AnykD polyacrylamide gels (BioRad) and transferred to nitrocellulose membranes by electroblotting. Following heating at $100^{\circ} \mathrm{C}$ in PBS for 25 seconds, membranes were blocked with $5 \%$ bovine serum albumin (BSA) in tris-buffered saline (TBS) and incubated with IrDye 680 streptavidin (diluted $1: 3,000$; LI-COR). Biotin-labeled polypeptides were visualized by nearinfrared fluorescence with an Odyssey imager as described ${ }^{32}$. The polypeptide input was verified in each experiment by parallel analysis of the same samples with either a rabbit anti-APP C-terminus antibody (for AICD-59), the $6 \mathrm{E} 10 \mathrm{mAb}$ (for $\mathrm{A} \beta_{42}$ ), or a rabbit anti-GST antibody (for GST-CTF $\alpha$ and GST).

Chromatin Immunoprecipitation assays. Chromatin immunoprecipitation (ChIP) assays were performed with the Magna ChIP Tissue kit (\#17-20000, Millipore). Tenmonth-old Tg2576 female mice (Taconic), fed with CHF5074-supplemented (375 ppm) or standard diet ( $n=6$ animals/group) were employed for these analyses. Animal care and treatments were in accordance with European Community Council directives (86/609/EEC) and in conformity with protocols approved by ethical committee for animal experimentation of University of Bologna.

Following sacrifice and brain dissection, three randomly assorted pools of entorhinal cortices from each group were frozen and chopped into small pieces. 
Minced tissue was cross-linked with $1.5 \%$ formaldehyde for 10 minutes at $37^{\circ} \mathrm{C}$ and subsequently incubated for $5 \mathrm{~min}$ in a $0.15 \mathrm{M}$ glycine solution to stop the reaction. Cross-linked tissue was washed with ice-cold PBS and incubated on ice for 15 minutes in Tissue Lysis Buffer. Nuclei were then pelleted ( $800 \mathrm{~g}, 5$ minutes at $4{ }^{\circ} \mathrm{C}$ ), resuspended in Dilution Buffer and sonicated. After centrifugation, aliquots of each chromatin sample were separately incubated with anti-APP C-terminus, anti-acetyl H3 (\#07-593; Millipore) or anti-IgG (negative control) antibodies. Magnetic protein $\mathrm{G}$ beads were then added, followed by overnight incubation at $4{ }^{\circ} \mathrm{C}$ on a rotating wheel. Another chromatin sample, not incubated with any antibody, served as input control. Antibody-captured protein/DNA complexes were washed, eluted and subjected to quantitative real-time polymerase chain reaction (qRT-PCR) analysis with the following forward (Fw) and reverse (Rev) primers: KAI1-Fw $5^{\prime}$-ATCATGCCATGCTGTCTGTC-3'; KAI1-Rev 5'-GGGCATAGGGTAGGCTGAG-3'

Immunoprecipitated DNA ( $2 \mu \mathrm{l} /$ sample) was amplified in a $25 \mu \mathrm{l}$ reaction mixture containing the SYBR Green master mix (BioRad). Real-time PCR analysis was carried out in triplicate using an iCycler (BioRad) apparatus. $\mathrm{C}_{\mathrm{T}}$ values generated by $\mathrm{qRT}$ PCR analysis of samples immunoprecipitated with the anti-APP C-terminus or antiacetyl-H3 antibodies were first normalized with respect to the corresponding $\mathrm{C}_{\mathrm{T}}$ values obtained from IgG-immunoprecipitated, negative control samples, and then with respect to the relative $\mathrm{C}_{\mathrm{T}}$ values obtained from "input" (i.e., "no antibody") chromatin samples.

Transcript level determinations. Total RNA was extracted from mouse entorhinal cortices of $\operatorname{Tg} 2576$ mice treated with CHF5074-supplemented or standard diet as described above ( $n=3$ animals/group) using the RNeasy Lipid Tissue Mini Kit (Qiagen, \# 74804). RNA (1 $\mu \mathrm{g} / \mathrm{sample}$ ) was reverse-transcribed with the Quantitect ${ }^{\circledR}$ Reverse Transcription Kit (Qiagen, \# 205313), using an optimized mix of oligo-dT, random primers and primer plus $7 \times$ gDNA Wipeout Buffer, and incubated at $42^{\circ} \mathrm{C}$ for 2 minutes to remove contaminating genomic DNA. $5 \times$ Quantiscript RT Buffer, Quantiscript Reverse Transcriptase and RT Primer Mix were then added and the reaction mixture was incubated at $42^{\circ} \mathrm{C}$ for 15 minutes, followed by thermal inactivation at $95^{\circ} \mathrm{C}$ for 3 minutes.

After cDNA synthesis, RT-PCR was carried out as described above, using the following KAI1 gene-specific primers $(0.3 \mathrm{pmol} / \mu \mathrm{L}): F w 5^{\prime}$-TTACGTCTTCATC GGTGTGG-3'; Rev 5' - CACTGTGTTCCCCATCTCCT-3'. The comparative $\mathrm{C}_{\mathrm{T}}$ method was used for relative mRNA quantification; $\beta$-actin, amplified with the $F w 5^{\prime}$ GGCTCTTTTCCAGCCTTCCT- $3^{\prime}$ and Rev $5^{\prime}$ - ATGCCTGGGTACATGGTGGT$3^{\prime}$ primers, was used for expression level normalization.

Statistical analysis. Statistical significance of differences between groups was evaluated by two-way ANOVA and Dunnet's multiple comparison tests using the GraphPad Prism 5 software (GraphPad Software, La Jolla, CA, USA). ChIP data were analyzed with Student's $t$-tests for independent data. $P$-values $(p)$ lower than 0.05 were considered as statistically significant.

1. LaFerla, F. M. \& Oddo, S. Alzheimer's disease: Abeta, tau and synaptic dysfunction. Trends Mol. Med. 11, 170-6 (2005).

2. Selkoe, D. J. Alzheimer's disease: genes, proteins, and therapy. Physiol. Rev. 81, 741-66 (2001)

3. Beckett, C., Nalivaeva, N. N., Belyaev, N. D. \& Turner, A. J. Nuclear signalling by membrane protein intracellular domains: the AICD enigma. Cell. Signal. 24 402-9 (2012).

4. Takami, M. \& Funamoto, S. gamma-Secretase-Dependent Proteolysis of Transmembrane Domain of Amyloid Precursor Protein: Successive Tri- and Tetrapeptide Release in Amyloid beta-Protein Production. Int. J. Alzheimers Dis. 2012, 591392 (2012)

5. Zettl, H., Weggen, S., Schneider, P. \& Schneider, G. Exploring the chemical space of gamma-secretase modulators. Trends Pharmacol. Sci. 31, 402-10 (2010).

6. Haapasalo, A. \& Kovacs, D. M. The many substrates of presenilin/gammasecretase. J. Alzheimers Dis. 25, 3-28 (2011).

7. Belyaev, N. D. et al. The transcriptionally active amyloid precursor protein (APP) intracellular domain is preferentially produced from the 695 isoform of APP in a beta-secretase-dependent pathway. J. Biol. Chem. 285, 41443-54 (2010).

8. Goodger, Z. V. et al. Nuclear signaling by the APP intracellular domain occurs predominantly through the amyloidogenic processing pathway. J. Cell Sci. 122, 3703-14 (2009).

9. Ghosal, K. et al. Alzheimer's disease-like pathological features in transgenic mice expressing the APP intracellular domain. Proc. Natl. Acad. Sci. U. S. A. 106, 18367-72 (2009)

10. Konietzko, U. AICD nuclear signaling and its possible contribution to Alzheimer's disease. Curr. Alzheimer Res. 9, 200-16 (2012).

11. Buoso, E., Lanni, C., Schettini, G., Govoni, S. \& Racchi, M. beta-Amyloid precursor protein metabolism: focus on the functions and degradation of its intracellular domain. Pharmacol. Res. 62, 308-17 (2010).

12. Kimberly, W. T., Zheng, J. B., Guenette, S. Y. \& Selkoe, D. J. The intracellular domain of the beta-amyloid precursor protein is stabilized by Fe65 and translocates to the nucleus in a notch-like manner. J. Biol. Chem. 276, 40288-92 (2001).

13. Kinoshita, A., Whelan, C. M., Smith, C. J., Berezovska, O. \& Hyman, B. T. Direct visualization of the gamma secretase-generated carboxyl-terminal domain of the amyloid precursor protein: association with Fe65 and translocation to the nucleus J. Neurochem. 82, 839-47 (2002).

14. von Rotz, R. C. et al. The APP intracellular domain forms nuclear multiprotein complexes and regulates the transcription of its own precursor. J. Cell Sci. 117, 4435-48 (2004).

15. Baek, S. H. et al. Exchange of N-CoR corepressor and Tip60 coactivator complexes links gene expression by NF-kappaB and beta-amyloid precursor protein. Cell 110, 55-67 (2002).

16. Cao, X. \& Sudhof, T. C. A transcriptionally [correction of transcriptively] active complex of APP with Fe65 and histone acetyltransferase Tip60. Science 293, 115-20 (2001).

17. Kim, H. S. et al. C-terminal fragments of amyloid precursor protein exert neurotoxicity by inducing glycogen synthase kinase-3beta expression. Faseb J. 17, 1951-3 (2003).

18. Hebert, S. S. et al. Regulated intramembrane proteolysis of amyloid precursor protein and regulation of expression of putative target genes. EMBO Rep. 7, 739-45 (2006).

19. Ryan, K. A. \& Pimplikar, S. W. Activation of GSK-3 and phosphorylation of CRMP2 in transgenic mice expressing APP intracellular domain. J. Cell Biol. 171, 327-35 (2005).

20. Telese, F. et al. Transcription regulation by the adaptor protein Fe65 and the nucleosome assembly factor SET. EMBO Rep. 6, 77-82 (2005).

21. Ghosal, K., Stathopoulos, A. \& Pimplikar, S. W. APP intracellular domain impairs adult neurogenesis in transgenic mice by inducing neuroinflammation. PLoS One 5, e11866 (2010).

22. Passer, B. et al. Generation of an apoptotic intracellular peptide by gammasecretase cleavage of Alzheimer's amyloid beta protein precursor. J. Alzheimers Dis. 2, 289-301 (2000).

23. Muller, T., Meyer, H. E., Egensperger, R. \& Marcus, K. The amyloid precursor protein intracellular domain (AICD) as modulator of gene expression, apoptosis, and cytoskeletal dynamics-relevance for Alzheimer's disease. Prog. Neurobiol. 85, 393-406 (2008).

24. Pardossi-Piquard, R. \& Checler, F. The physiology of the beta-amyloid precursor protein intracellular domain AICD. J. Neurochem. 120 Suppl 1, 109-24 (2012).

25. Imbimbo, B. P. et al. CHF5074, a novel gamma-secretase modulator, attenuates brain beta-amyloid pathology and learning deficit in a mouse model of Alzheimer's disease. Br. J. Pharmacol. 156, 982-93 (2009).

26. Lanzillotta, A. et al. The gamma-secretase modulator CHF5074 reduces the accumulation of native hyperphosphorylated tau in a transgenic mouse model of Alzheimer's disease. J. Mol. Neurosci. 45, 22-31 (2011).

27. Imbimbo, B. P. et al. CHF5074, a novel gamma-secretase modulator, restores hippocampal neurogenesis potential and reverses contextual memory deficit in a transgenic mouse model of Alzheimer's disease. J. Alzheimers Dis. 20, 159-73 (2010).

28. Sivilia, S. et al. Multi-target action of the novel anti-Alzheimer compound CHF5074: in vivo study of long term treatment in Tg2576 mice. BMC Neurosci. 14, 44 (2013).

29. Ross, J. et al. CHF5074 Reduces Biomarkers of Neuroinflammation in Patients with Mild Cognitive Impairment: A 12-Week, Double-Blind, Placebo- Controlled Study. Curr. Alzheimer Res. 10, 742-53 (2013).

30. Imbimbo, B. P. et al. 1-(3',4'-Dichloro-2-fluoro[1,1' -biphenyl]-4-yl)cyclopropanecarboxylic acid (CHF5074), a novel gamma-secretase modulator, reduces brain beta-amyloid pathology in a transgenic mouse model of Alzheimer's disease without causing peripheral toxicity. J. Pharmacol. Exp. Ther. 323, 822-30 (2007)

31. Imbimbo, B. P. et al. Pharmacokinetics and Pharmacodynamics of CHF5074 After Short-term Administration in Healthy Subjects. Alzheimer Dis. Assoc Disord. 27, 278-86 (2013).

32. Balducci, C. et al. The gamma-secretase modulator CHF5074 restores memory and hippocampal synaptic plasticity in plaque-free Tg2576 mice. J. Alzheimers Dis. 24, 799-816 (2011).

33. Giuliani, A. et al. CHF5074 restores visual memory ability and pre-synaptic cortical acetylcholine release in pre-plaque Tg2576 mice. J. Neurochem. 124, 613-20 (2013).

34. Dovey, H. F. et al. Functional gamma-secretase inhibitors reduce beta-amyloid peptide levels in brain. J. Neurochem. 76, 173-81 (2001).

35. Kukar, T. L. et al. Substrate-targeting gamma-secretase modulators. Nature 453, 925-9 (2008).

36. Golde, T. E., Koo, E. H., Felsenstein, K. M., Osborne, B. A. \& Miele, L. gammaSecretase inhibitors and modulators. Biochim. Biophys. Acta (2013).

37. Takami, M. et al. gamma-Secretase: successive tripeptide and tetrapeptide release from the transmembrane domain of beta-carboxyl terminal fragment. J. Neurosci. 29, 13042-52 (2009).

38. Kinoshita, A., Whelan, C. M., Berezovska, O. \& Hyman, B. T. The gamma secretase-generated carboxyl-terminal domain of the amyloid precursor protein induces apoptosis via Tip60 in H4 cells. J Biol Chem 277, 28530-6 (2002).

39. Cao, X. \& Sudhof, T. C. Dissection of amyloid-beta precursor protein-dependent transcriptional transactivation. J. Biol. Chem. 279, 24601-11 (2004).

40. Barbagallo, A. P. et al. $\operatorname{Tyr}(682)$ in the intracellular domain of APP regulates amyloidogenic APP processing in vivo. PLoS One 5, e15503 (2010). 
41. Xie, Z., Dong, Y., Maeda, U., Xia, W. \& Tanzi, R. E. RNA interference silencing of the adaptor molecules ShcC and Fe65 differentially affect amyloid precursor protein processing and Abeta generation. J. Biol. Chem. 282, 4318-25 (2007)

42. Galvan, V. et al. Reversal of Alzheimer's-like pathology and behavior in human APP transgenic mice by mutation of Asp664. Proc. Natl. Acad. Sci. U. S. A. 103, 7130-5 (2006).

43. Lichtenstein, M. P. et al. Secretase-independent and RhoGTPase/PAK/ERKdependent regulation of cytoskeleton dynamics in astrocytes by NSAIDs and derivatives. J. Alzheimers Dis. 22, 1135-55 (2010).

44. Muller, T. et al. Modulation of gene expression and cytoskeletal dynamics by the amyloid precursor protein intracellular domain (AICD). Mol. Biol. Cell 18, 201-10 (2007).

45. Ma, H. et al. Involvement of beta-site APP cleaving enzyme 1 (BACE1) in amyloid precursor protein-mediated enhancement of memory and activity-dependent synaptic plasticity. Proc. Natl. Acad. Sci. U. S. A. 104, 8167-72 (2007)

46. Belyaev, N. D., Nalivaeva, N. N., Makova, N. Z. \& Turner, A. J. Neprilysin gene expression requires binding of the amyloid precursor protein intracellular domain to its promoter: implications for Alzheimer disease. EMBO Rep. 10, 94-100 (2009)

47. Pimplikar, S. W. \& Suryanarayana, A. Detection of APP intracellular domain in brain tissue. Methods Mol. Biol. 670, 85-91 (2011).

48. Winkler, E. et al. Purification, pharmacological modulation, and biochemical characterization of interactors of endogenous human gamma-secretase. Biochemistry 48, 1183-97 (2009).

49. Rubio, I. et al. Potent anti-HPV immune responses induced by tandem repeats of the HPV16 L2 (20 -- 38) peptide displayed on bacterial thioredoxin. Vaccine 27, 1949-56 (2009).

\section{Acknowledgments}

This work was supported by a grant from the National Institute of Neuroscience to M.P. and a grant from the Regione Emilia-Romagna (Project: "Innovative technologies for the causal therapy of neurodegenerative diseases”; DGR n. 1631/2009) to S.O. and L.C.

\section{Author contributions}

C.B., I.S., A.L., M.B. and V.P. performed A $\beta$ peptide determinations, immunoblotting and photolabeling analyses on whole cell extracts, membrane and nuclear fractions from H4swe cells and ChIP assays. R.R. and A.R.V. produced the recombinant GST-CTF $\alpha$ fusion polypeptide and carried out photoaffinity labeling experiments on the AICD (poly)peptides. L.L. and L.C. took care of Tg2576 mice handling and treatment. B.P.I. contributed to data analysis. S.O. and M.P. designed the experiments and wrote the paper

\section{Additional information}

Supplementary information accompanies this paper at http://www.nature.com/ scientificreports

Competing financial interests: This study was supported in part by Chiesi Farmaceutici, Parma, Italy. Bruno P. Imbimbo is an employee of Chiesi Farmaceutici. The other authors declare no conflict of interest.

How to cite this article: Branca, C. et al. Pharmacological targeting of the $\beta$-amyloid precursor protein intracellular domain. Sci. Rep. 4, 4618; DOI:10.1038/srep04618 (2014).

This work is licensed under a Creative Commons Attribution-NonCommercialNoDerivs 3.0 Unported License. The images in this article are included in the article's Creative Commons license, unless indicated otherwise in the image credit; if the image is not included under the Creative Commons license, users will need to obtain permission from the license holder in order to reproduce the image. To view a copy of this license, visit http://creativecommons.org/licenses/by-nc-nd/3.0/ 